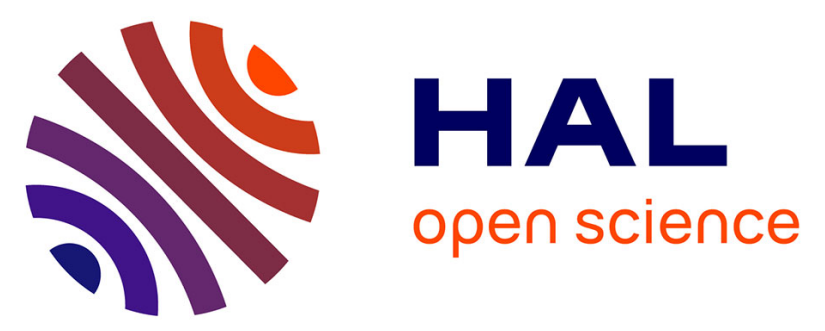

\title{
Transmission electron microscopy characterization of low temperature boron doped silicon epitaxial films
}

\author{
Guillaume Noircler, Marta Chrostowski, Melvyn Larranaga, Etienne Drahi, \\ Pere Roca I Cabarrocas, Patricia de Coux, Bénédicte Warot-Fonrose
}

\section{- To cite this version:}

Guillaume Noircler, Marta Chrostowski, Melvyn Larranaga, Etienne Drahi, Pere Roca I Cabarrocas, et al.. Transmission electron microscopy characterization of low temperature boron doped silicon epitaxial films. CrystEngComm, 2020, 22 (33), pp.5464-5472. 10.1039/D0CE00817F . hal-03030275

\section{HAL Id: hal-03030275 \\ https://hal.science/hal-03030275}

Submitted on 14 Dec 2020

HAL is a multi-disciplinary open access archive for the deposit and dissemination of scientific research documents, whether they are published or not. The documents may come from teaching and research institutions in France or abroad, or from public or private research centers.
L'archive ouverte pluridisciplinaire HAL, est destinée au dépôt et à la diffusion de documents scientifiques de niveau recherche, publiés ou non, émanant des établissements d'enseignement et de recherche français ou étrangers, des laboratoires publics ou privés. 


\section{Transmission Electron Microscopy Characterization of Low Temperature Boron Doped Silicon Epitaxial Films}

Guillaume NOIRCLER ${ }^{*, 1,2,3}$, Marta CHROSTOWSKI ${ }^{1,3,4}$, Melvyn LARRANAGA ${ }^{2}$, Etienne DRAHI ${ }^{1,3}$, Pere ROCA i CABARROCAS ${ }^{3,4}$, Patricia de COUX ${ }^{1}$, and Bénédicte WAROT-FONROSE ${ }^{2}$

${ }^{1}$ Total S.A., 2 place Jean Millier, 92400 Paris La Défense, France

${ }^{2}$ CEMES-CNRS, Université de Toulouse, CNRS, 29 rue Jeanne Marvig, 31055 Toulouse, France

${ }^{3}$ Institut Photovoltaïque d'Ile-de-France (IPVF), 18, Boulevard Thomas Gobert, 91120 Palaiseau France

${ }^{4}$ LPICM-CNRS - Ecole Polytechnique, Institut Polytechnique de Paris, 91128 PALAISEAU, France

*Corresponding author: guillaume.noircler@cemes.fr 


\section{Abstract}

Transmission Electron Microscopy (TEM) techniques can provide a complementary understanding of the physico-chemical mechanisms of the growth and the annealing behavior of boron-doped hydrogenated silicon epitaxial films grown at low temperature $\left(<200^{\circ} \mathrm{C}\right)$ by Radio Frequency Plasma Enhanced Chemical Vapor Deposition (RF-PECVD). Compared to the standard boron diffusion or ion implantation, the PECVD process ensures a lower thermal budget and the realization of a sharper doping profile. The complexity of this epi-layer process lies on the fact that many impurities are incorporated during growth and their analysis requires the use of advanced characterization methods. Particular attention is paid to defects by combining High Resolution Transmission Electron Microscopy (HRTEM) and an image processing routine called Geometric Phase Analysis (GPA) which allows to study strain field. For the as-grown epitaxial layer, we will see how the non-conventional PECVD growth process influences its microstructure and gives to it a non-uniform strain-field. In the annealed epitaxial layer, no strain is measured but nanotwins have been detected and analyzed through a geometric model. Scanning TEM (STEM) techniques are also applied to analyze chemical variation at the interface. A darker contrast is observed at the interface of the annealed sample most likely due to the migration of hydrogen and oxygen atoms during annealing.

\section{Introduction}

Solar cells based on crystalline silicon nearly reach the theoretical efficiency limit with a record of $26,7 \%$ [1]. They are the basis of a fairly mature and affordable technology representing over $90 \%$ of commercial photovoltaic panels [2]. Materials investigation could lead to a better understanding of their properties and innovation in manufacturing processes to build new structures like silicon/perovskite tandem cell, still with the aim of increasing the production yield and lower their cost. Low temperature plasma-enhanced chemical vapor deposition (PECVD) is an alternative way to form p-n junction for solar cells. Compared to the standard boron diffusion which requires high processing and/or annealing temperatures, increasing the overall thermal budget of the fabrication and the risk of wafer damage, due to impurities diffusion and thermal donor activation, low temperature $\left(<200^{\circ} \mathrm{C}\right) \mathrm{PECVD}$ of boron doped silicon ensures a lower thermal budget, the simplification of the process flow by reducing the number of fabrication steps and the realization of a sharper and much more controllable doping profile. 
Boron doped silicon epitaxy is therefore proposed in order to replace the standard boron rear emitter diffusion for n-PERT technology. More recently, it has also been investigated as a way to grow tunnel junctions for two-terminal (2-T) perovskite/silicon tandem cells. The low temperature PECVD growth process for these silicon epitaxial films implies the formation of silicon-hydrogen $\left(\mathrm{Si}_{n} \mathrm{H}_{m}\right)$ nanocrystals in the plasma that are accelerated towards the substrate and melt upon impact[3][4][5]. This type of growth is completely different from the well known PECVD growth process of silicon films which is achieved through the reaction between the substrate surface and $\mathrm{SiH}_{3}$ radicals [6][7]; yet both starting with a $\mathrm{SiH}_{4}$ plasma. In this paper, the boron doped silicon epitaxy, already characterized by X-Ray Diffraction (XRD), Ellipsometry, Secondary Ion Mass Spectrometry (SIMS), electrochemical capacitance voltage (ECV) and photoluminescence [8][9][10], is studied at local scale by using TEM techniques in order to bring new relevant information about the growth process and to understand the impact of annealing on the boron-doped epi-si layer grown at $175^{\circ} \mathrm{C}$. Indeed, while the previously cited techniques give indirect and macroscopic physico-chemical information, TEM techniques provide direct and local information thanks to their atomic scale resolution. TEM enables to appreciate the microstructure of the layer particularly at the interface that is known to be critical for the performance of the device. It has already been used for low temperature silicon epitaxy to characterize mostly the crystalline quality [11][12][13] or defects analysis such as H-platelets [14]. In this article, several TEM techniques were applied both to complete Chrostowski characterization [8][9] and to provide new keys to understand better the epitaxy morphology and the annealing effect. Particular attention was paid to defects and strain in the layer and at the interface with a different approach from what has been done so far by combining HRTEM post-treatment, STEM and geometric/crystallographic models. Therefore, this investigation will help to identify the parameters or process steps to optimize.

\section{Experimental}

The p+ epi-layers were grown by Chrostowski et al. [8][9] in a semi-industrial capacitively coupled radio-frequency PECVD reactor at a process temperature of $175^{\circ} \mathrm{C}$ from a $\mathrm{H}_{2} / \mathrm{SiH}_{4} / \mathrm{TMB}$ (trimethylboron, $\left.\left(\mathrm{CH}_{3}\right)_{3} \mathrm{~B}\right)$ gas mixture on phosphorus-doped (100) double-side polished Float Zone (FZ) crystalline silicon wafers. The latter were dipped into $5 \%$ HF to remove native oxide and allow epitaxial growth. After this process, the epi-layer is annealed in forming gas $\left(5 \% \mathrm{H}_{2}\right.$ in $\left.\mathrm{N}_{2}\right)$ during 10 minutes at $350^{\circ} \mathrm{C}$. For further details on the process, refer to [8][9]. In this paper, two samples were studied, 
one with the as-grown epitaxial layer (referred as "as-grown epi-layer") and one with the annealed layer (referred as " $350^{\circ} \mathrm{C}$-annealed epi-layer"). Those two samples have been characterized by XRD, Ellipsometry and SIMS, the characterization results are summarized in Table 1.

Table 1: Chrostowski et al. characterization summary [8][9]

\begin{tabular}{clcc}
\hline & & As-grown & $\mathbf{3 5 0}^{\circ} \mathbf{C}$-annealed \\
\hline \multicolumn{1}{c}{ XRD } & Out-of-plane lattice & $\mathrm{a}_{\perp}{ }^{*}=5.4437 \AA\left(>\mathrm{a}_{\mathrm{s}}\right)^{*}$ & $\mathrm{a}_{\perp}=\mathrm{a}_{\mathrm{s}}$ \\
& $\begin{array}{l}\text { parameter } \\
\text { Nature of the strain }\end{array}$ & Fully strained & Fully relaxed \\
\hline Ellipsometry & Crystallinity & Between poly-Si and c-Si & Closer to c-Si \\
& Thickness & $195 \mathrm{~nm}$ & $165 \mathrm{~nm}$ \\
\hline ECV + SIMS & Doping efficiency & $0 \%$ & $50 \%$ \\
\hline${ }^{*}$ Out-of-plane lattice parameter & & \\
${ }^{* *}$ c-Si substrat lattice parameter & &
\end{tabular}

TEM requires specific sample preparation to the materials and TEM characterization techniques before using it for analysis. By using Focused Ion Beam (FIB), electron transparent lamella were lifted out as cross sections through the epi-layer using an adaptation of the Schaffer et al. method [15]. This FIB lift out technique allows to have the least damage of the sample and to have the best atomic resolution in STEM and HRTEM. For this, we used an FEI Helios NanoLab 600i. The first step of the FIB preparation is the orientation of the sample to have the desired [110] zone axis to control the growth direction [001] of the epitaxy and to observe low index family planes edge on. Then, a protection layer of the sample surface was deposited with electron beam and ion beam platinum deposition. Initial wedge-milling was performed using a decrease Gallium ions voltage from $30 \mathrm{kV}$ to $5 \mathrm{kV}$ with the cleaning cross section (CCS) pattern. The thinning was carried out in staircase profile, each voltage corresponding to a step of the stair ; this allows us to see the progress of the thinning. Once the $5 \mathrm{kV}$ of ion beam was reached, we used electron beam at $5 \mathrm{kV}$ during milling to monitor the thinning process. A contrast reversal allows us to monitor the thickness of the lamella. The last thinning steps were done at $2 \mathrm{kV}$ and $1 \mathrm{kV}$ with Rectangular Cross Section pattern and the thickness monitoring with electron beam at $2 \mathrm{kV}$. This technique is much longer (around 4 hours) than the classic one but gives high quality and thin $(\leq 90 \mathrm{~nm})$ electron transparent area over $6 \mu \mathrm{m}$ length for (S)TEM analysis. This method was chosen because silicon FZ here used as a substrate is more likely 
to be contaminated than silicon Czochralski. Indeed, the increased purity of the material leads to a larger sensitivity to contamination. Thus, this is a real challenge both for preparation (ion beam) and for observation (electron beam and carbon contamination). In this article, two samples were prepared with the same method to have similar thickness (determined by Electron Energy Loss Spectroscopy (EELS) to be homogeneous at $90 \mathrm{~nm}$ for both samples) to be properly compared: as-grown and $350^{\circ} \mathrm{C}$-annealed epi-layers.

High Resolution Transmission Electron Microscopy (HRTEM) characterizations were done by using a spherical aberration-corrected transmission electron microscope (SACTEM) based on a 200-kV Tecnai F-20 (FEI) with a maximum resolution of $0.1 \mathrm{~nm}$. This microscopy technique allows to have structural contrasts to study the crystalline quality and the defects at the atomic level. HRTEM low magnification images were done at first to have an overview of the microstructure of the epi-layer over the $6 \mu \mathrm{m}$ long lamella. Secondly, high magnification images were made to analyze the contrast and the defects around the interface and in the epi-layer. By using HRTEM images and Geometric Phase Analysis (GPA), it is possible to map strain field [16]. GPA is indeed an image processing technique capable of resolving lattice strain according to the analysis of local maxima (atoms) contrast in the image. For this purpose, a mask is applied to the Fourier transform of the HRTEM image and the inverse-Fourier transform is performed. The resulting phase image is related to the displacement field where a reference lattice must be defined. In our case we took the reference in c-Si substrate. Then GPA goes up to the full deformation tensor. In this article we will be interested in the tension/compression components of the strain $\epsilon[17]$.

JEOL ARM200F spherical aberration-corrected TEM with a maximum resolution of $0.08 \mathrm{~nm}$ was used for chemical imaging and spectroscopy. STEM high-angle annular dark-field (STEM-HAADF) image gives a contrast depending approximately on the square of the atomic number $\mathrm{Z}^{2}$ and on the specimen thickness hence the importance of preparing two specimens with the same thickness so that they are perfectly comparable. We also used EELS, exploiting the fact that incident electrons loose energy when they pass through the sample, this energy loss is measured with an EEL spectrometer and the spectra provide information on the chemical composition of the materials [18]. Two distinct parts of the spectra can be exploited: the low loss part related to the optical and/or magnetic properties, and the high loss part for chemical composition or oxidation degree. Here we used the low loss only for specimen thickness determination and the high loss to analyze if there is any change in chemical composition at the interface. 


\section{Results}

As presented above, ellipsometry and XRD bring macroscopic information about the crystallinity and the strain in the epi-layer. HRTEM images and a specific post-treatment called Geometric Phase Analysis (GPA) will complete and bring direct additional local structural information about defects, strain and chemical variations.
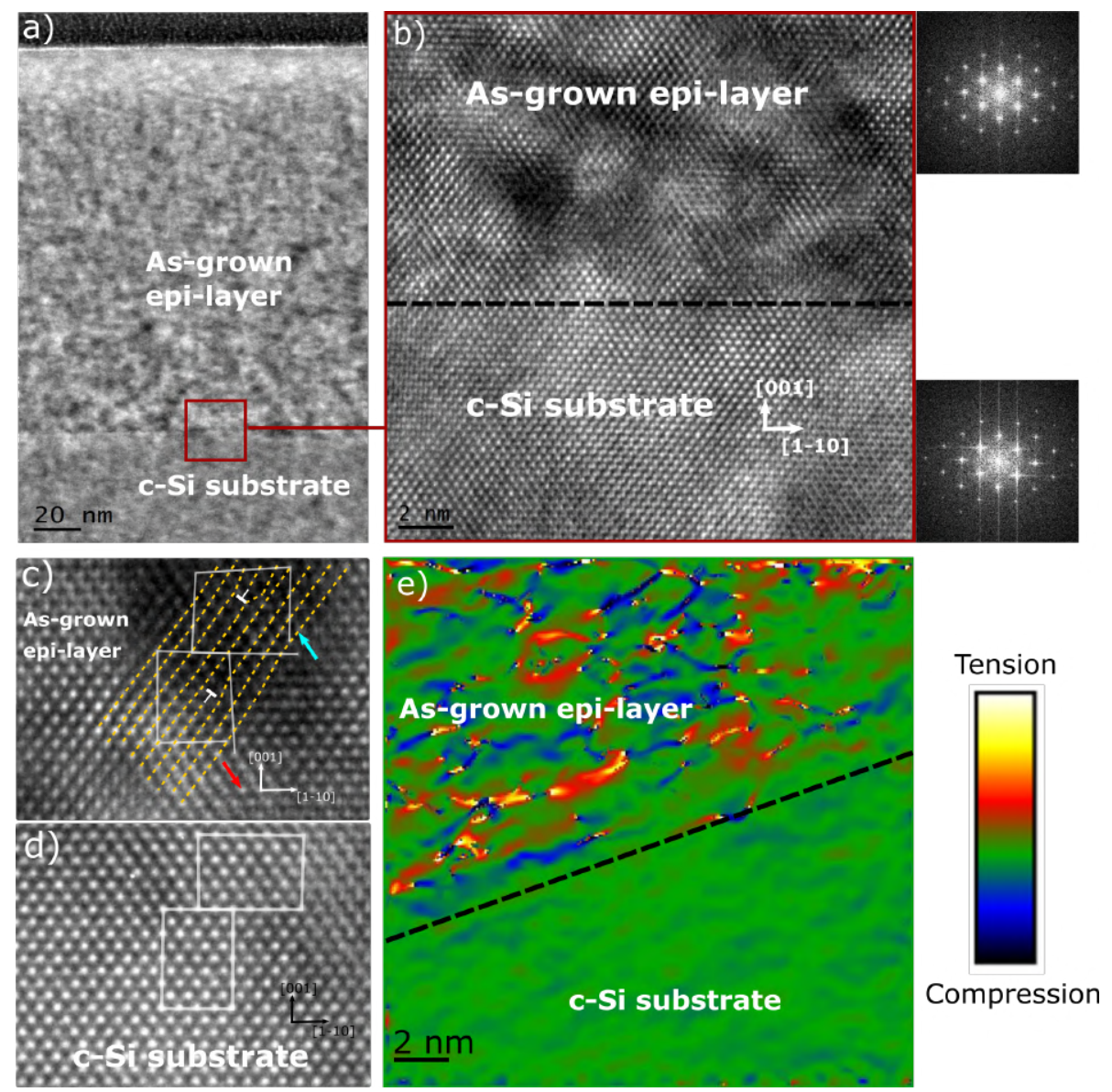

Figure 1: Low a) and High b) magnification HRTEM images of as-grown epi-layer. c) HRTEM image showing two dislocations using a Burgers circuit (drawn in white), Burgers vectors are represented by the two blue and red arrows and the orange doted lines evidence (1-1-1) planes that helps to visualize the dislocations. d) Same Burgers circuits are closed when drawn on perfect Si crystal. e) GPA out-of-plane strain field mapping showing a large density of dislocations and other defects.

Figure 1 a) and b) shows low and high magnification HRTEM images for the as-grown epi-layer. Low magnification image (Figure 1.a)) reveals a very flat and well defined film. Still, from low magnification image, the epi-layer can be easily identified with respect to the substrate, which makes thickness 
measurement easier both for ellipsometry and HRTEM. HRTEM thickness measurements of $198 \mathrm{~nm}$ for the as-grown, and of $163 \mathrm{~nm}$ for the $350^{\circ} \mathrm{C}$-annealed epi-layer are in agreement with the thickness given in Table 1. The as-grown epi-layer contrast is completely different from that originating from the substrate. The epi-layer exhibits round black and white contrasts which will be referred as speckled contrast in this article. Those contrasts are homogeneously distributed along all the $6 \mu \mathrm{m}$ as-grown FIB-sample preparation of the epi-layer. From the bottom to the top of the film, three parts can be distinguished: (1) coarse speckled constrast, (2) more fine speckled contrast, (3) only white contrast. In the high magnification image (Figure 1.b)) around the interface, we observe that despite those contrasts which probably result from a large amount of strain, the crystalline structure propagates from the wafer to the epi-layer. Furthermore, the fast Fourier transforms (FFT) corresponding to the substrate and the epi-layer are the same, which means that the crystalline quality of the epi-layer is comparable to the substrate. We found some dislocations in the epi-layer which are located both in the layer and at the interface. Figure 1.c) shows two of these dislocations located in the film. Burgers circuits have been drawn first in the substrate Figure 1.d) and secondly in the epi-layer Figure 1.c) to point out closure defects induced by each dislocation. We found that the total Burgers vector of those dislocation can be either (1/2)[10-1] or (1/2)[0-1-1] for the red Burgers vector Figure 1.c) and $(1 / 2)[-101]$ or $(1 / 2)[011]$ for the blue Burgers vector Figure 1.c) (those Burgers vectors are given in c-Si lattice parameter unit). These Burgers vectors have a component on which the sign is unknown because it is parallel to the [110] zone axis. Both of these mixed dislocations belong to the (1-11) glide plane which is a compact plane of the structure. In other words, they could contribute to the relaxation of the lattice submitted to an external constraint (thermal or mechanical). In Figure 1.e), GPA was applied on HRTEM images to map strain field in the epi-layer and around the interface with respect to a reference area chosen in the silicon substrate. Figure 1.e) shows that as-grown epi-layer has a non-uniform strain field probably due to high density of defects, this will be discussed in next section. In other words, the strain in the layer is distributed in many nano-regions (less than $3 \mathrm{~nm}$ ). Some nano-regions are highly strained in compression or in tension while others present no strain compared to the c-Si substrate reference. 

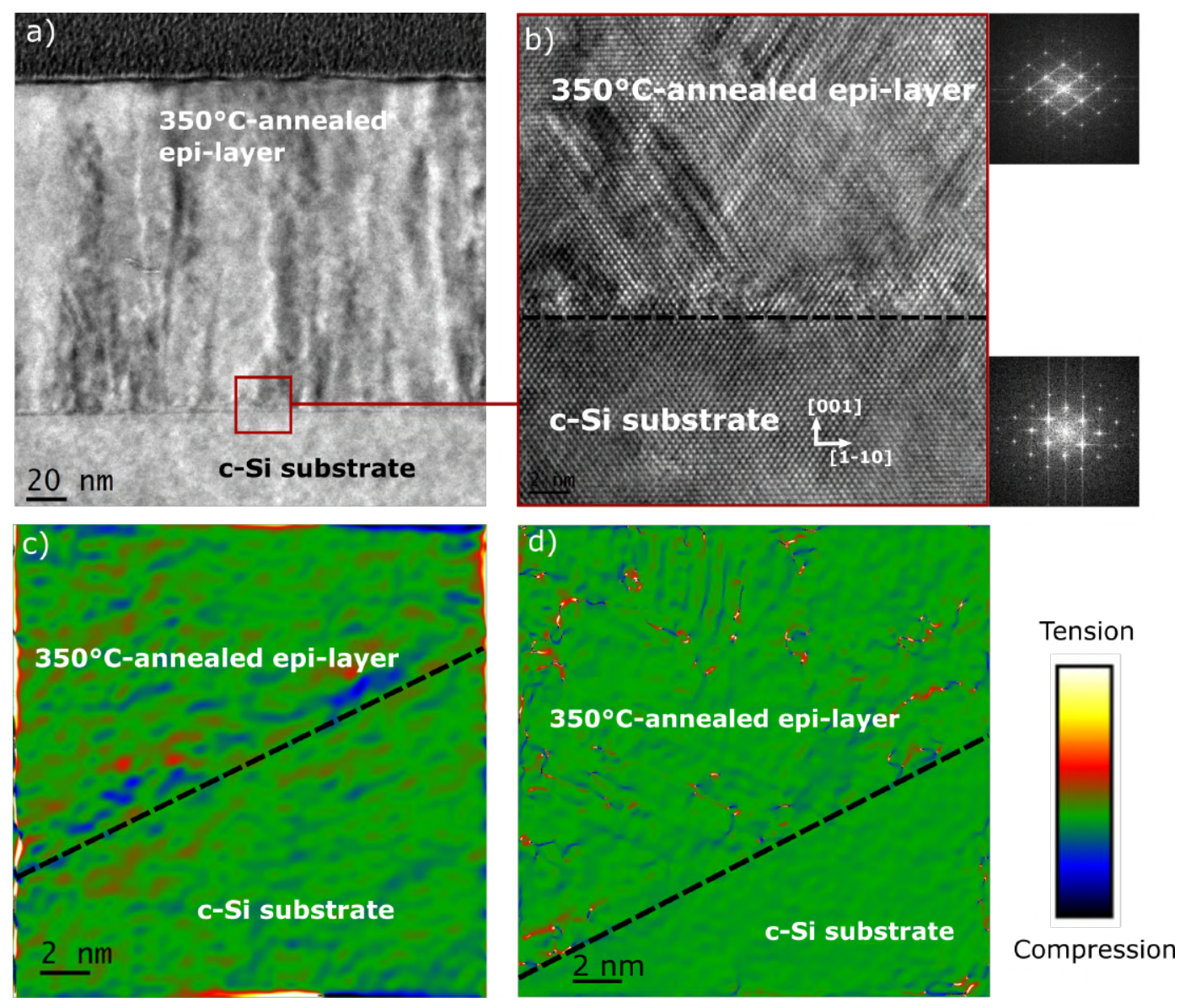

Figure 2: Low a) and High b) magnification HRTEM images of the $350^{\circ} \mathrm{C}$-annealed epi-layer sample. c) and d) GPA out-of-plane strain-field mapping in area without nanotwin and with nanotwins respectively.

Figure 2 presents the $350^{\circ} \mathrm{C}$-annealed sample analyzed with the same HRTEM protocol at low (Figure 2.a)) and high (Figure 2.b)) magnification images and FFT of HRTEM images. A completely different morphology is noticed compared to the as-grown epi-layer; the speckled contrast has indeed totally disappeared. This annealed film shows a huge heterogeneity in its micro-structure: while some parts have the same contrast as the substrate, other parts have columnar defects that begin at the interface and can continue to the surface or either stop in the film or continue in another direction that is not visible in the micrography. Outside the columnar defects, a high magnification image with FFT near the interface shows that the epi-layer has substrate crystalline quality. Inside the columnar defects there are some V-shaped defects that lie on $\{111\}$ type planes. In the latter area, the FFT exhibit some streaks along $\{111\}$ spots which are characteristic of nanotwinning [19]. In Figure 2.c) and d), we applied GPA inside and outside the columnar defects of the $350^{\circ} \mathrm{C}$-annealed epi-layer. On 
average $0 \%$ strain was measured in both cases. However, in Figure 2.d), some very local combination of tension/compression strain that are characteristic of dislocations are highlighted by GPA. Finally, the epi-layer has changed from as-grown with a wide distribution of strain and defects to a fully relaxed epi-layer with some dislocations.

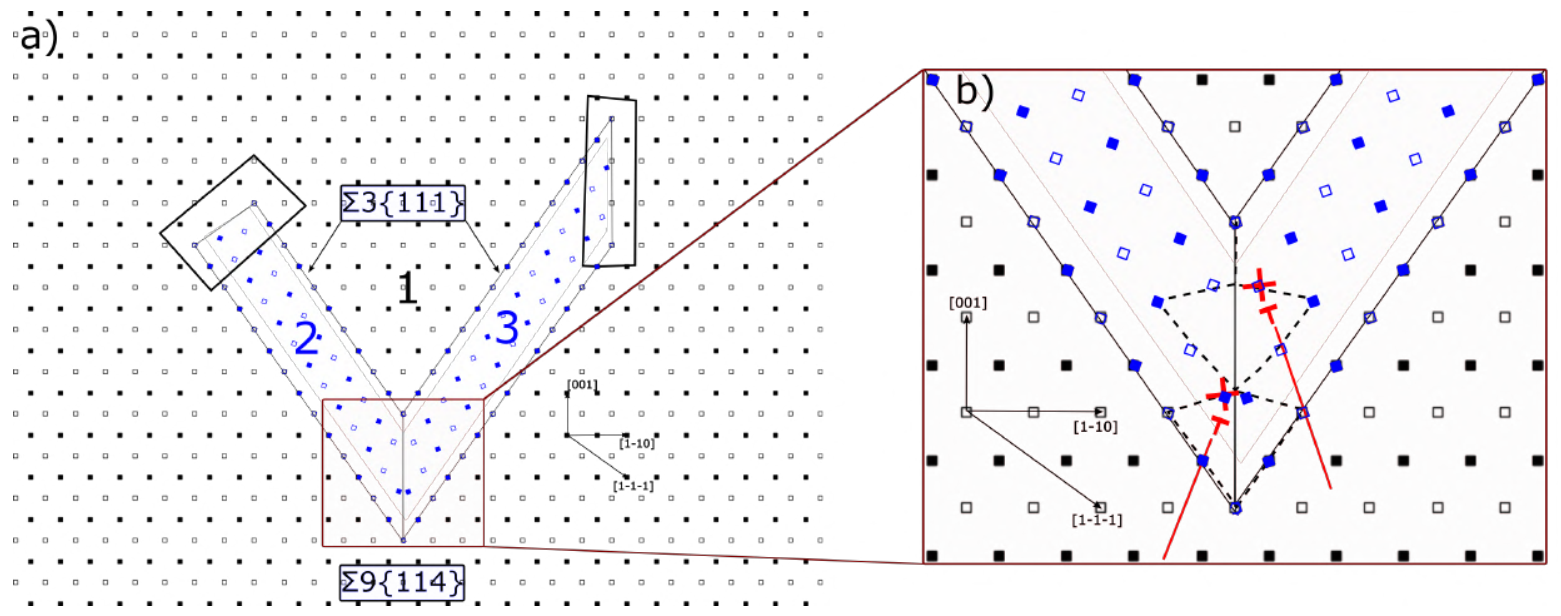

Figure 3: a) Nanotwin model showing its atomic structure and evidencing the needed GBs between the twinned lattices (2 and 3 ) and the matrix (1). According to this model, dislocations might appear in order to accommodate the misorientation between the lattices (framed areas). b) Inset showing a possible dislocation structure for the $\Sigma 9\{114\}$ GB between the twinned lattices.

Figure 3 shows a simple geometrical model of the possible structure of the observed nanotwins. Two types of twin boundary planes, $(-111)_{1}$ and $(1-11)_{1}$ are necessary for recovering the shape of the nanotwin and this can be readily obtained by considering two opposite rotations of the matrix lattice in order to generate the two twinned lattices. Those rotations are $(+/-) 70,53^{\circ}$ around [110] zone axis. Then each twinned ( 2 and 3 drawn in blue in Figure 3.a)) lattice is joined to the matrix lattice along the fully coincident (-111) and (1-11) planes that results in the formation of four $\Sigma 3\{111\}$ twin boundaries (TB). Thus the nanotwins are expected to be almost free of structural defects. However there are also areas where higher index grain boundaries (GBs) are needed and therefore structural dislocations may locally appear. On one hand, the closure of the nanotwin means that there are other grain boundaries with the matrix. From the image it is difficult to determine in which plane these GBs are localized. Moreover in this case there are multiple possibilities of grain boundary plane (framed in black in Figure 3.a)) and this geometrical model does not provide information about the relative energetic stability between these GBs. On the other hand, there is another GB between the two twinned lattices inside the nanotwin (framed in red in Figure 3.a). The orientation relationship 
between these lattices is also a special one that results from the coincidence of one site over nine and can be described as $\Sigma 938,94^{\circ}[110]$. In this case there is a mirror plane of these lattices, namely $(-114)_{2} /(-11-4)_{3}$, that would be an interesting GB plane as shown in Figure 3.a). We can notice that in this case of symmetric GB, dislocations may be needed as displayed in red in Figure 3.b).

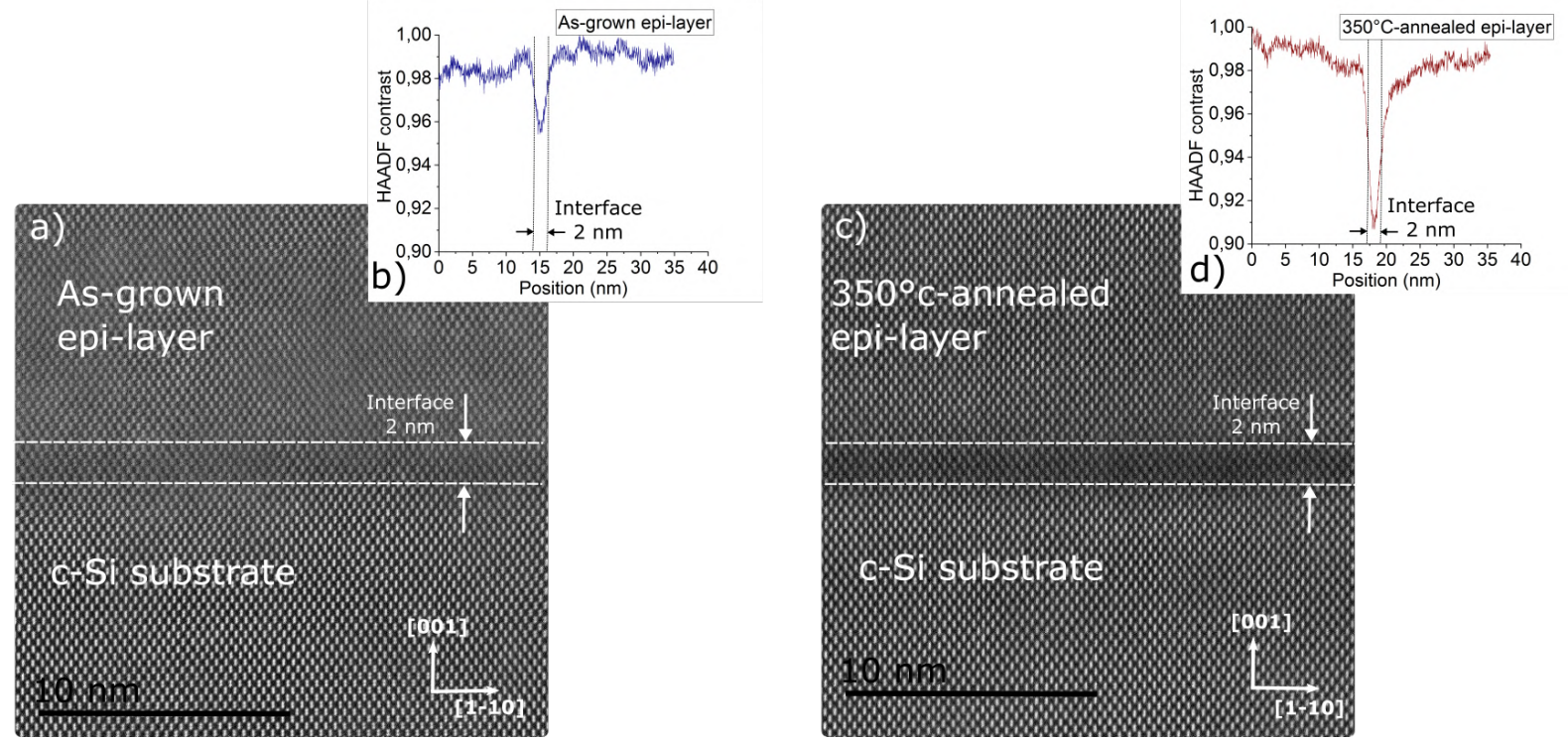

Figure 4: STEM-HAADF images and there atomic density profile. a)STEM-HAADF image with its associated b) contrast profile of the as-grown epi-layer. c)STEM-HAADF image with its associated d) contrast profile of the $350^{\circ} \mathrm{C}$-annealed epi-layer.

To complete the interface studies, STEM-HAADF was used to display chemical information. STEM-HAADF images (Figure 4) around the interface show for both states of the epi-layer a contrast of $2 \mathrm{~nm}$ at the interface. This contrast is more pronounced for the $350^{\circ} \mathrm{C}$-annealed epi-layer. The atomic density profile (normalized with respect to the substrate) is displayed for those two images. A density drop of $4.5 \%$ is detected for the as-grown epi-layer whereas $9 \%$ is measured for the $350^{\circ} \mathrm{C}$ annealed epi-layer. This indicates that the modification of the chemical composition is more important for the $350^{\circ} \mathrm{C}$-annealed epi-layer. To identify the origin of this contrast, we also made EELS localized at the interface. In EELS the number of atoms per unit area is proportional to the integrated intensity of each edges present in the spectrum. So, here the aim was to measure a local variation of the composition at the interface. To do so we searched for the oxygen and boron edge but nothing was detected indicating that the oxygen and boron concentration in the epi-layer and at the interface was below the detection limit of EELS. Nevertheless, the intensity of the Si edge decrease slightly at 
the interface which must be related to hydrogen or voids left behind after annealing and hydrogen out diffusion.

\section{Discussion}

The speckled contrast that exhibits the as-grown epi-layer has already been observed in intrinsic epi-layers grown by low temperature PECVD which contain a large amount of Hydrogen $\left([\mathrm{H}]>10^{20}\right.$ at $\left./ \mathrm{cm}^{3}\right)$ [20] [21] [14] [22][13]. This contrast was characterized as hydrogen-platelets. H-platelet can be described as a micro-crack in the crystal that separates two or more adjacent planes. The latter occurs especially on $\{100\}$ and $\{111\}$ familly planes and contains H-terminated silicon and molecular Hydrogen[23][24][25][26]. In TEM by tilting the sample and with a contrast reversal upon changing from under-focus to over-focus, an H-platelet appears as 2D plate-like structure which can be seen edge-on or not [14]. In the edge-on specific case, it appears like a stick, otherwise it displays a round contrast due to the associated strain field [22]. In our case, even if the layer presents this characteristic contrast, a conventional TEM experiment shows no H-platelets. Furthermore, the zone axis [110] that we choose for HRTEM images permits to display the $\{001\}$ and $\{111\}$ family planes nonetheless we never observed H-platelets edge-on lying on them. Later in the discussion, we will see that hydrogen has a very great affinity for boron, much greater than for silicon[27][28]. This would therefore explain the fact that there is no or such a small amount of $\mathrm{H}$-platelets that we were unable to observe them in the epi-layer.

GPA measurements (Figure 1.e)) show a large heterogeneity of the out-of-plane strain field. Some nano-regions are compressively or tensively strained or present no strain. A previous XRD experiment on the as-grown epi-layer shows that the layer is fully strained (the in-plane lattice parameter of the layer is strained to be identical to that of the substrate) and that there is an increase of the outof-plane lattice parameter of $0.24 \%$ with respect to the substrate (Table 1). The broad FWHM of the $\omega / 2 \theta$ peak of the as-grown epi-layer also indicates that the out-of-plane increase is heterogeneous [8]. GPA measurements are in agreement with XRD results, and provide complementary information about the defects distribution. Here we can say that some defects homogeneously distributed in the layer have generated non-uniform strain field, and have lead to an increase of the avarage out-of-plane lattice parameter. To understand this let us consider the PECVD growth mechanism. Indeed, as we said before, $\mathrm{Si}_{n} \mathrm{H}_{m}$ nano-clusters formed in the plasma contribute to the formation of the epi-layer. 
The growth of the epitaxial film depends on the impact energy of these clusters when they hit the substrate. As soon as they get in touch with the latter one, a local phase transition occurs at very high temperature (around $3000 \mathrm{~K}$ ) which lead to the melting of the $\mathrm{Si}_{n} \mathrm{H}_{m}$ nanocrystals followed by their thermalisation with the substrate[5][29]. If we extended this reasoning for the growth of the entire epitaxy, each nano-clusters would hit each other with a certain amount of energy leading each time to a phase transition followed by their thermalisation generating a certain amount of local strain and lattice mismatch (hence the presence of dislocations Figure 1.c)), this is therefore what GPA treatment has highlighted in Figure 1.e. In other words, we believe that these defects or nano-regions that create the non-uniform strain field measured in GPA are related to the organization of the $\mathrm{Si}_{n} \mathrm{H}_{m}$ nano-clusters during the epitaxial growth process.

Previous ECV measurements [8] on the as-grown epi-layer have reported no doping efficiency indicating that boron atoms are incorporated at interstitial position(Table 1). With these results and HRTEM findings, we might think at first that boron atoms are in interstitial position. In the case of high hydrogen incorporation in the film (here $[\mathrm{H}]=2.10^{21} \mathrm{at} / \mathrm{cm}^{3}$ ) with the presence of boron (here $\left.[\mathrm{B}]=3.10^{19} \mathrm{at} / \mathrm{cm}^{3}\right)$, it is known that hydrogen can neutralize $99 \%$ of boron atoms by forming B-H complexes [27][28]. In the B-H model complex of Herrero et al. [28], $\mathrm{H}$ is in tetrahedral interstitial site of the $\mathrm{Si}$ and neutralizes the boron atoms that are on substitutional sites. They also show that those B-H complexes induce a lattice dilatation which is in agreement with XRD measurements. Once the totality of boron atoms are neutralized by hydrogen, the quantity of hydrogen remaining can combine with other impurities such as carbon or oxygen. C-H interstitial link to substitutional Carbon were already characterized in c-Si by luminescence [30] for c-Si annealed at $400^{\circ} \mathrm{C}-600^{\circ} \mathrm{C}$, however $\mathrm{C}-\mathrm{H}$ complexes dissociate and become electrically neutral at room temperature [31]. Concerning O-H complexes, they are also unstable at room temperature but it is well known that Hydrogen facilitates oxygen migration and enhances the formation of oxygen aggregates [32][33]. This phenomenon could explain the darker STEM-HAADF contrast (Figure 4) at the interface for both as-grown and annealed epi-layer and also the the greater oxygen concentration around the interface measured by SIMS for the as-grown epi-layer [9]. Another candidate for the source of defects in our silicon epitaxy could be oxygen due its abnormal high concentration with respect to the substrate probably due to some outgassing in the reactor. Silicon oxide precipitates have specific morphology (platelike, spherical, octahedral) and are often observed after heat treatment at high temperature $\left(400-1200^{\circ} \mathrm{C}\right)$ [34] [35]. None of those silicon oxide precipitates were found in the as-grown epi-layer. However the abnormal oxygen 
concentration may lead to high concentration of interstitial oxygen which can form electrically active donors thus reducing the acceptor density in the p-type as-grown epitaxy. Finally for the as-grown epi-layer, we believe that mostly B-H complexes, oxygen aggregates and interstitial, are incorporated in the epi-layer, and they could also be responsible of the non-conductivity of the as-grown epi-layer. Besides, the broad peak around $0.9 \mathrm{eV}$ that has been detected in Photoluminescence by Chrostowski et al. [9] and interpreted as strain around extended hydrogen-defects by Weman et al. [36] could be related to $\mathrm{B}-\mathrm{H}$ complexes in $\mathrm{Si}_{n} \mathrm{H}_{m}$ nano-clusters. In addition, the interference fringes at low energy in ellipsometry [9] indicates a defective interface composed of a mix between voids, silicon oxide and c-Si depending on the fitting model used. The drop in the atomic density profile (Figure 4.b) confirms that the interface is composed of elements other than silicon. For the as-grown epi-layer, we thought that the interface is most likely composed by c-Si, residual native oxide and interstitial oxygen atoms as explained before.

The annealing at $350^{\circ} \mathrm{C}$ has a strong impact on the morphology and strain field, indeed it heals the micro-structure by considerably reducing the density of defects and relaxing the strain. The speckled contrast described before completely disappeared after annealing. This could be a signature of hydrogen desorption and so a total reorganization of the crystal whose main building blocks were previously the $\mathrm{Si}_{n} \mathrm{H}_{m}$ nano-clusters as well as the breaking of the B-H complexes which blocked the conductivity. Actually, a doping efficiency of $50 \%$ was measured after annealing. At this point, the question is: Why don't we have $100 \%$ ? In attempting to answer this question let us discuss the morphology and the relaxation of strain in the annealed epi-layer. Houben et al. [37] also noticed columnar defects in micro-crystalline silicon and found that the columnar growth results from the formation of nanotwins which is in agreement with our HRTEM analysis. Haddad et al. [38] also observed nanotwins in intrinsic silicon epitaxy either just before epitaxy breakdown or before epitaxy restoration. Thus, annealing induces a reorganization of the crystal by the formation of nanotwins. We can also deduce from GPA results that annealing gives some energy to the epi-layer which has relaxed through the formation of structural defects like nanotwins which begin at the interface and propagate through the film via columnar defects to relax the strain in the epitaxial layer. XRD and ellipsometry measurements on this layer also proved a relaxation of the strain and an improvement of the crystalline quality respectively. Regarding the presence of twinning in the layer, it has been stated by Möller [39] that beyond $10^{18}$ at $/ \mathrm{cm}^{3}$ of carbon impurities, they accumulate on $\{111\}$ planes inducing many twin boundaries. This phenomenon would therefore not be surprising given the amount of carbon $\left([\mathrm{C}]=3.10^{19} \mathrm{at} / \mathrm{cm}^{3}\right)$ in our 
layer. Grain boundaries and dislocations are generally electrically active and they are also known as segregation centers for impurities, therefore they are always undesirable. $\Sigma 3\{111\}$ TBs were characterized to be electrically inactive by Chen et al. [40] using Electron Beam Induced current (EBIC) that is to say they do not induce any recombination activity. Moreover, Ohno et al. [41] have also shown by Atom Probe Tomography (APT) coupled with TEM that TB has a weak intrinsic segregating ability even for a high concentration of impurities. However, Sarau et al. [42], have measured by EBIC that TB can be either recombination-free or recombination active depending on the presence of dislocations around the GB. $\Sigma 9\{114\}$ contains intrinsic dislocations as shown in Figure 3. Moreover, it has been proved by Ohno et al. [43] using APT and TEM that a large amount of oxygen can segregate at this GB. Electrical activity and segregation at GB are complex topics which depend on dislocations, impurity concentration and decoration, intrinsic structure, type, density and spatial arrangement around and inside the GB. A key to a better understanding of GB electrical/recombination activity in our epitaxial layer would be EBIC and/or Cathodoluminescence in a TEM. This leads us to conclude that the areas presenting nanotwins might result in a loss of conductivity. Furthermore STEM-HAADF images (Figure 4.c) and d) analysis show that the modification of the chemical composition at the interface is even more important for the $350^{\circ}$-annealed epi-layer than for the as-grown one. Indeed, the annealing probably promotes the migration of hydrogen and/or oxygen atoms at the interface, which could be another source of degradation of electronic properties.

\section{Conclusion}

Adding (S)TEM investigation to the characterization of boron-doped hydrogenated silicon epitaxial layers grown at low temperature allowed to make further headway in understanding structural features generated by growth and annealing. Firstly, HRTEM has shown that despite defects in the epitaxy, the crystalline structure propagates from the substrate to the epi-layer for both as-grown and $350^{\circ} \mathrm{C}$ annealed epi-layers. Secondly, HRTEM coupled with GPA has highlighted a non-uniform strain field induced by the impact of silicon clusters, which are the building blocks of the as-grown epi-layer. The annealing impact on the epi-layer has been shown to reduce the density of defects and to induce a restructuring of the epi-layer through the formation of nanotwins which relax the strain in the epilayer. By studying the structure of nanotwins through a geometric model, we determine that they are formed by four $\Sigma 3\{111\}$ twin boundaries and one $\Sigma 9\{114\}$ GB. We have shown that the latter defects 
could limit the doping efficiency and so the conductivity of the epi-layer.

\section{References}

[1] Kunta Yoshikawa et al. "Silicon Heterojunction Solar Cell with Interdigitated Back Contacts for a Photoconversion Efficiency over 26\%". en. In: Nature Energy 2.5 (Mar. 2017), pp. 1-8. ISSN: 2058-7546. DOI: $10.1038 /$ nenergy $\cdot 2017.32$.

[2] Lucio Claudio Andreani et al. "Silicon Solar Cells: Toward the Efficiency Limits". In: Advances in Physics: X 4.1 (Jan. 2019), p. 1548305. ISSN: null. DOI: 10.1080/23746149.2018.1548305.

[3] P. Roca i Cabarrocas, R. Cariou, and M. Labrune. "Low Temperature Plasma Deposition of Silicon Thin Films: From Amorphous to Crystalline". en. In: Journal of Non-Crystalline Solids 358.17 (Sept. 2012), pp. 2000-2003. ISSN: 00223093. DOI: 10.1016/j.jnoncrysol.2011.12.113.

[4] P. Roca i Cabarrocas et al. "Low Temperature Plasma Synthesis of Nanocrystals and Their Application to the Growth of Crystalline Silicon and Germanium Thin Films". en. In: MRS Proceedings 1426 (2012), pp. 319-329. ISSN: 0272-9172, 1946-4274. DOI: 10.1557/opl.2012.1094.

[5] Ha-Linh Thi Le, Fatme Jardali, and Holger Vach. "Deposition of Hydrogenated Silicon Clusters for Efficient Epitaxial Growth". en. In: Physical Chemistry Chemical Physics 20.23 (2018), pp. 15626-15634. ISSN: 1463-9076, 1463-9084. DOI: 10.1039/C8CP00764K.

[6] W.M.M. Kessels et al. "On the Growth Mechanism of A-Si:H". en. In: Thin Solid Films 383.1-2 (Feb. 2001), pp. 154-160. ISSN: 00406090. DOI: 10.1016/S0040-6090(00)01594-7.

[7] Takuya Kuwahara et al. "The Reason Why Thin-Film Silicon Grows Layer by Layer in PlasmaEnhanced Chemical Vapor Deposition". en. In: Scientific Reports 5.1 (Mar. 2015), pp. 1-7. ISSN: 2045-2322. DOI: $10.1038 /$ srep09052.

[8] Marta Chrostowski et al. "Low Temperature Epitaxial Growth of Boron-Doped Silicon Thin Films". In: SILICONPV 2018, THE 8TH INTERNATIONAL CONFERENCE ON CRYSTALLINE SILICON PHOTOVOLTAICS. Lausanne, Switzerland, 2018, p. 070001. DOI: 10 . $1063 / 1$. 5049300.

[9] Marta Chrostowski et al. "Annealing of Boron-Doped Hydrogenated Crystalline Silicon Grown at Low Temperature by PECVD". en. In: Materials 12.22 (Jan. 2019), p. 3795. DOI: 10.3390/ ma12223795. 
[10] M. Labrune, M. Moreno, and P. Roca i Cabarrocas. "Ultra-Shallow Junctions Formed by QuasiEpitaxial Growth of Boron and Phosphorous-Doped Silicon Films at $175^{\circ} \mathrm{C}$ by Rf-PECVD". en. In: Thin Solid Films 518.9 (Feb. 2010), pp. 2528-2530. ISSN: 00406090. DOI: 10.1016/j.tsf . 2009.09.143.

[11] Ronan Léal et al. "High Quality Boron-Doped Epitaxial Layers Grown at $200^{\circ} \mathrm{C}$ from $\mathrm{SiF}_{4} / \mathrm{H}_{2}$ /Ar Gas Mixtures for Emitter Formation in Crystalline Silicon Solar Cells". en. In: AIP Advances 7.2 (Feb. 2017), p. 025006. ISSN: 2158-3226. DOI: 10.1063/1.4976685.

[12] Romain Cariou. "Epitaxial Growth of $\mathrm{Si}(\mathrm{Ge})$ Materials on $\mathrm{Si}$ and GaAs by Low Temperature PECVD: Towards Tandem Devices". Thesis. Palaiseau, Ecole polytechnique, Jan. 2014.

[13] Wanghua Chen et al. "Influence of Deposition Rate on the Structural Properties of PlasmaEnhanced CVD Epitaxial Silicon”. en. In: Scientific Reports 7.1 (Apr. 2017), p. 43968. ISSN: 2045-2322. DOI: $10.1038 /$ srep43968.

[14] G. B. Anderson, C. C. Tsai, and R. Thompson. "Transmission Electron Microscopy of HydrogenInduced Defects in Low Temperature Epitaxial Silicon". en. In: MRS Online Proceedings Library Archive 262 (1992/ed). ISSN: 0272-9172, 1946-4274. DOI: 10.1557/PROC-262-241.

[15] Miroslava Schaffer, Bernhard Schaffer, and Quentin Ramasse. "Sample Preparation for AtomicResolution STEM at Low Voltages by FIB". en. In: Ultramicroscopy 114 (Mar. 2012), pp. 62-71. ISSN: 03043991. DOI: 10.1016/j.ultramic.2012.01.005.

[16] M. J. Hÿtch, E. Snoeck, and R. Kilaas. "Quantitative Measurement of Displacement and Strain Fields from HREM Micrographs". en. In: Ultramicroscopy 74.3 (Aug. 1998), pp. 131-146. ISSN: 0304-3991. DOI: 10.1016/S0304-3991 (98)00035-7.

[17] C. Gatel et al. "Magnetism of CoFe2O4 Ultrathin Films on MgAl2O4 Driven by Epitaxial Strain". In: Applied Physics Letters 103.9 (Aug. 2013), p. 092405. ISSN: 0003-6951. DOI: 10 .1063/1. 4819178.

[18] R.F. Egerton and M. Malac. "EELS in the TEM". en. In: Journal of Electron Spectroscopy and Related Phenomena 143.2-3 (May 2005), pp. 43-50. ISSN: 03682048. DOI: 10.1016/j .elspec. 2003.12 .009 .

[19] C. Cayron et al. "Odd Electron Diffraction Patterns in Silicon Nanowires and Silicon Thin Films Explained by Microtwins and Nanotwins". en. In: Journal of Applied Crystallography 42.2 (Apr. 2009), pp. 242-252. ISSN: 0021-8898. DOI: 10.1107/S0021889808042131. 
[20] N. M. p Johnson et al. "Defects in Single-Crystal Silicon Induced by Hydrogenation". In: Physical Review B 35.8 (Mar. 1987), pp. 4166-4169. DOI: 10.1103/PhysRevB.35.4166.

[21] Romain Cariou et al. "Low Temperature Plasma Enhanced CVD Epitaxial Growth of Silicon on GaAs: A New Paradigm for III-V/Si Integration". en. In: Scientific Reports 6.1 (Sept. 2016). ISSN: 2045-2322. DOI: $10.1038 /$ srep25674.

[22] C. C. Tsai, G. B. Anderson, and R. Thompson. "Low Temperature Growth of Epitaxial and Amorphous Silicon in a Hydrogen-Diluted Silane Plasma”. In: Journal of Non-Crystalline Solids 137-138 (Jan. 1991), pp. 673-676. ISSN: 0022-3093. DOI: 10.1016/S0022-3093(05)80210-8.

[23] Peter Deák et al. "Structure of the (1 11 1) Hydrogen Platelet in Silicon". In: Physica B: Condensed Matter 170.1 (Apr. 1991), pp. 223-226. ISSN: 0921-4526. DOI: 10.1016/0921-4526 (91) 90126-Y.

[24] Gianpietro Moras et al. "Atomically Smooth Stress-Corrosion Cleavage of a Hydrogen-Implanted Crystal". In: Physical Review Letters 105.7 (Aug. 2010), p. 075502. DOI: 10.1103/PhysRevLett. 105.075502.

[25] S. Muto, S. Takeda, and M. Hirata. "Hydrogen-Induced Platelets in Silicon Studied by Transmission Electron Microscopy". In: Philosophical Magazine A 72.4 (Oct. 1995), pp. 1057-1074. ISSN: 0141-8610. DOI: 10.1080/01418619508239953.

[26] T. Akatsu et al. "Study of Extended-Defect Formation in Ge and Si after H Ion Implantation". In: Applied Physics Letters 86.18 (Apr. 2005), p. 181910. ISSN: 0003-6951. DOI: 10.1063/1.1906319.

[27] N. M. Johnson. "Mechanism for Hydrogen Compensation of Shallow-Acceptor Impurities in Single-Crystal Silicon”. In: Physical Review B 31.8 (Apr. 1985), pp. 5525-5528. DoI: 10.1103/ PhysRevB.31.5525.

[28] C. P. Herrero, M. Stutzmann, and A. Breitschwerdt. "Boron-Hydrogen Complexes in Crystalline Silicon". In: Physical Review B 43.2 (Jan. 1991), pp. 1555-1575. DOI: 10.1103/PhysRevB. 43. 1555.

[29] G. Hamon et al. "Heteroepitaxial Growth of Silicon on GaAs via Low-Temperature PlasmaEnhanced Chemical Vapor Deposition". In: Quantum Sensing and Nano Electronics and Photonics XVI. Vol. 10926. International Society for Optics and Photonics, Feb. 2019, p. 109261C. DOI: $10.1117 / 12.2511174$.

[30] A. N. Safonov et al. "Interstitial-Carbon Hydrogen Interaction in Silicon". In: Physical Review Letters 77.23 (Dec. 1996), pp. 4812-4815. DOI: 10.1103/PhysRevLett.77.4812. 
[31] A. L. Endrös, W. Krühler, and F. Koch. "Electronic Properties of the Hydrogen-carbon Complex in Crystalline Silicon". In: Journal of Applied Physics 72.6 (Sept. 1992), pp. 2264-2271. Issn: 0021-8979. DOI: $10.1063 / 1.351620$.

[32] A. R. Brown et al. "Enhanced Thermal Donor Formation in Silicon Exposed to a Hydrogen Plasma". en. In: Semiconductor Science and Technology 3.6 (June 1988), pp. 591-593. ISSN: 0268-1242. DOI: $10.1088 / 0268-1242 / 3 / 6 / 013$.

[33] H. J. Stein and S. K. Hahn. "Hydrogen-accelerated Thermal Donor Formation in Czochralski Silicon". In: Applied Physics Letters 56.1 (Jan. 1990), pp. 63-65. ISSN: 0003-6951. DOI: 10.1063/ 1.102652.

[34] C. Claeys, E. Simoen, and J. Vanhellemont. "Electrical and Structural Properties of OxygenPrecipitation Induced Extended Defects in Silicon". In: Journal de Physique III 7.7 (July 1997), pp. 1469-1486. ISSN: 1155-4320, 1286-4897. DOI: 10.1051/jp3:1997200.

[35] J. Nicolai et al. "Characterizing and Modeling the Evolution of Silicon Oxide Precipitates during Thermal Cycles". en. In: Journal of Crystal Growth 372 (June 2013), pp. 138-144. ISSN: 00220248. DOI: $10.1016 / j \cdot j$ jcrysgro.2013.03.041.

[36] H. Weman, J. L. Lindström, and G. S. Oehrlein. "Photoluminescence of Defects Introduced by Deuterium Plasmas in Silicon”. en. In: Materials Science and Engineering: B 4.1 (Oct. 1989), pp. 461-465. ISSN: 0921-5107. DOI: 10.1016/0921-5107(89)90287-0.

[37] L. Houben, M. Luysberg, and R. Carius. "Microtwinning in Microcrystalline Silicon and Its Effect on Grain-Size Measurements". en. In: Physical Review B 67.4 (Jan. 2003), p. 045312. ISSN: 0163-1829, 1095-3795. DOI: 10.1103/PhysRevB.67.045312.

[38] Farah Haddad. "Transmission Electron Microscopy Study of Low-Temperature Silicon Epitaxy by Plasma Enhanced Chemical Vapor Deposition". Thesis. Paris Saclay, Dec. 2016.

[39] Hans Joachim Möller. Carbon-Induced Twinning in Multicrystalline Silicon. en. 2004. DOI: 10 . 4028/wWw. scientific.net/SSP. 95-96.181.

[40] Jun Chen et al. "Recombination Activity of $\Sigma 3$ Boundaries in Boron-Doped Multicrystalline Silicon: Influence of Iron Contamination”. In: Journal of Applied Physics 97.3 (Dec. 2004), p. 033701. ISSN: 0021-8979. DOI: 10.1063/1.1836009. 
[41] Yutaka Ohno et al. "Three-Dimensional Evaluation of Gettering Ability of $\Sigma 3\{111\}$ Grain Boundaries in Silicon by Atom Probe Tomography Combined with Transmission Electron Microscopy". In: Applied Physics Letters 103.10 (Sept. 2013), p. 102102. ISSN: 0003-6951. DOI: 10.1063/1. 4820140.

[42] G. Sarau et al. "Correlating Internal Stresses, Electrical Activity and Defect Structure on the Micrometer Scale in EFG Silicon Ribbons". en. In: Solar Energy Materials and Solar Cells. Photovoltaics, Solar Energy Materials \& Thin Films, IMRC 2009-Cancun 95.8 (Aug. 2011), pp. 2264-2271. ISSN: 0927-0248. DOI: 10.1016/j. solmat.2011.03.039.

[43] Y. Ohno et al. "Nanoscopic Analysis of Oxygen Segregation at Tilt Boundaries in Silicon Ingots Using Atom Probe Tomography Combined with TEM and Ab Initio Calculations". en. In: Journal of Microscopy 268.3 (2017), pp. 230-238. ISSN: 1365-2818. DOI: 10.1111/jmi.12602. 